\title{
The Pleotrophic Effects of Insulin-Like Growth Factor-I on Human Spinal Cord Neural Progenitor Cells
}

\author{
J. Simon Lunn,, Crystal Pacut,, Carey Backus, ${ }^{1}$ Yu Hong, ${ }^{1}$ Karl Johe, ${ }^{2}$ Michael Hefferan, ${ }^{3}$ \\ Martin Marsala, ${ }^{3}$ and Eva L. Feldman ${ }^{1}$
}

\begin{abstract}
Most stem cell therapies involve direct, intraparachymal placement of neural progenitor cells. These cells provide physical support to the endogenous neuronal population and may be engineered to provide in situ growth factor support. Insulin-like growth factor-I (IGF-I) has potent neurotrophic and neuroprotective properties and is expressed by human neural stem cells (hNSCs). IGF-I is implicated in multiple aspects of cell behavior, including proliferation, differentiation, and survival. Enhancing hNSC function through IGF-I overexpression may increase the benefits of stem cell therapy. As a first step to that goal, we examined the direct effects of IGF-I on hNSC behavior in vitro. We demonstrate that IGF-I treatment enhances both the number and length of hNSC neurites. This is correlated with a decrease in proliferation, suggesting that IGF-I promotes neurite outgrowth but not proliferation. While IGF-I activates both AKT and MAPK signaling in hNSCs, we demonstrate that IGF-I-mediated neurite outgrowth is dependent only on AKT signaling. Finally, we demonstrate that IGF-I is neuroprotective after glutamate exposure in a model of excitotoxic cell death.
\end{abstract}

\section{Introduction}

$\mathrm{H}$ UMAN NEURAL STEM CELLS (hNSCs) derived from fetal spinal cord differentiate into neurons that mature and integrate when implanted directly into rat spinal cord whether naive or injured by various means [1-4]. As hNSCs differentiate, they produce several trophic factors, including vascular endothelial growth factor (VEGF), brain-derived neurotrophic factor (BDNF), glial-cell-derived neurotrophic factor (GDNF), and insulin-like growth factor-I (IGF-I) [1]. Intraspinal injection of hNSCs into the gray matter reveals quantitative survival of the hNSC with limited cellular migration and a 3-4-fold increase in cell number. Three months after injection, the majority of grafted hNSCs in the spinal cord differentiate into TuJ1-positive neurons expressing neurotransmitter markers of both glutamatergic and GABAergic neurons, project extensive neurofilament (NF)positive axons into the host tissue, and form synapses with the host motor neurons (MNs) [1,2].

IGF-I is a potent trophic factor essential for both neuronal development and normal function [5,6]. IGF-I signaling through the type I IGF receptor (IGF-IR) stimulates proliferation, differentiation, neurite outgrowth, and survival of multiple cell types, including MNs and sensory neurons, oligodendrocytes, and Schwann cells [7-16]. Several groups have demonstrated the protective capacity of IGF-I in multiple cell types, including MNs $[11,15,17,18]$. Vincent et al. demonstrated that IGF-I protects primary MNs against glutamate excitotoxicity [11,19]. In vivo, IGF-I signaling is required in the developing cortex for axonal outgrowth [20]. When IGF-I-producing cells were grafted into the spinal cord after injury, in situ IGF-I production was sufficient to protect against neural degeneration and promote partial re-growth [21].

Advances in cellular therapies have led to the development of combinatorial strategies. Donor cells engineered to produce additional growth factors provide both cellular and trophic support as potential therapeutics [22-25]. For multifaceted neurodegenerative diseases such as amyotrophic lateral sclerosis (ALS) and Alzheimer's disease, cellular therapies may provide a possibility for therapeutic development circumventing unknown cellular mechanisms. Recent studies demonstrate the increased potency of neural precursors in conjunction with GDNF for MN support in ALS models $[26,27]$. Initial development of hNSC treatment for both spinal cord trauma and ALS has demonstrated their beneficial potential as a treatment option [2-4]. Currently, the hNSCs described here are the first cellular therapy approved for Phase 1 human trials in ALS due to their innate ability to protect MNs and effectively incorporate into the spinal cord.

This work was presented at the Society for Neuroscience Meeting, 2009, Chicago, under the title of "Functional Effects of IGF-I During the Differentiation of Human Spinal Cord Stem Cells."

${ }^{1}$ Department of Neurology, University of Michigan, Ann Arbor, Michigan.

${ }^{2}$ Neuralstem, Inc., Rockville, Maryland.

${ }^{3}$ Department of Anesthesia, University of California, San Diego, California. 
Enhancing hNSC function by adding additional trophic factor production may result in greater therapeutic potential.

As a first step toward studying the combination of IGF-I with hNSC therapy for neuronal injury, we investigated the direct effects of IGF-I on hNSC in chemically defined culture. We demonstrate that IGF-I has positive effects on both neuronal survival and axonal outgrowth without affecting proliferation or differentiation of hNSCs. Our findings suggest that a combination of IGF-I overexpression and hNSC may enhance stem cell therapy for the treatment of neurodegenerative diseases and neuronal injuries.

\section{Materials and Methods}

\section{Cell culture}

All culture reagents were obtained from Sigma unless otherwise noted. hNSCs were prepared from a cervicalupper thoracic region of spinal cord tissue obtained from a single 8-week human fetus after an elective abortion. The fetal tissue was donated by the mother in a manner fully compliant with the guidelines of NIH and FDA and approved by an outside independent review board as described [28]. Cell culture was carried out as described previously [1,3]. Briefly, flasks were prepared by incubation for $24 \mathrm{~h}$ at room temperature with $100 \mu \mathrm{g} / \mathrm{mL}$ poly-D-lysine (Millipore) in $10 \mathrm{mM}$ HEPES buffer at $0.165 \mathrm{~mL} / \mathrm{cm}^{2}$. The flasks were washed 3 times with water and allowed to completely dry aseptically in the hood. They were then further incubated with $25 \mu \mathrm{g} / \mathrm{mL}$ fibronectin/phosphate-buffered saline (PBS) for $1 \mathrm{~h}$. The fibronectin solution was aspirated, and the vessels were used immediately without drying. Cells were cultured in a growth medium supplemented with Dulbecco's modified Eagle's medium (DMEM)/F12 (Gibco BRL Invitrogen) supplemented with $100 \mathrm{mg} / \mathrm{L}$ human plasma apo-transferrin, $25 \mathrm{mg} / \mathrm{L}$ recombinant human insulin, $1.56 \mathrm{~g} / \mathrm{L}$ glucose, $20 \mathrm{nM}$ progesterone, $100 \mu \mathrm{M}$ putrescine, and $30 \mathrm{nM}$ sodium selenite. For growth of hNSCs and maintenance in a progenitor state $10 \mathrm{ng} / \mathrm{mL}$ fibroblast growth factor (FGF) was added to the growth medium. For differentiation, hNSCs were cultured in a differentiation medium: DMEM supplemented with $4 \mathrm{mM}$ L-glutamine, $20 \mu \mathrm{M}$ L-alanine, $6 \mu \mathrm{M}$ L-asparagine, $67 \mu \mathrm{M}$ L-proline, $250 \mathrm{nM}$ vitamin B12, $25 \mathrm{mg} / \mathrm{L}$ insulin, $100 \mathrm{mg} / \mathrm{L}$ transferring, $20 \mathrm{nM}$ progesterone, $100 \mu \mathrm{M}$ putrescine, and $30 \mathrm{nM}$ sodium selenite. The differentiation medium contains no FGF. The medium was changed every 2 days with a $50 \%$ medium change. hNSCs grown in the presence of IGF-I had IGF-I supplemented to a final concentration of $10 \mathrm{nM}$ at each medium change.

\section{Immunocytochemistry}

Immunocytochemistry (ICC) was performed as previously described [29]. Briefly, hNSCs were grown on poly-D-lysine and fibronectin-coated glass coverslips in 24-well plates, fixed with $4 \%$ paraformaldehyde (PFA), permeabilized with $0.1 \%$ Triton/PBS, and then blocked in 5\% normal donkey serum/0.1\% Triton/PBS. NF (1:1,000; Millipore), IGF-IR $\beta$ (1:500; Sigma), GluR2 (1:200; Abcam), VGlut2 (1:200; Millipore), GAD (1:200; Millipore), Synaptophysin (Syn, 1:200; Millipore), or TuJ1 (1:500, Neuromics Edina) primary antibodies were incubated overnight at $4{ }^{\circ} \mathrm{C}$. Cells were then incubated in Cy3, Cy5, or fluorescein-isothiocyanate-conjugated secondary antibodies (Jackson ImmunoResearch) followed by mounting on glass slides using ProLong Gold+DAPI (MolecularProbes, Invitrogen). Images were collected using an Olympus BX-51 microscope.

To measure proliferation, cells were grown as above and cells were incubated with $10 \mu \mathrm{M}$ 5'-ethynyl-2'-deoxyuridine (EdU) for $2 \mathrm{~h}$ before fixation and processing following the manufacturers' protocols for the Click-It EdU kit (Invitrogen). We measured EdU incorporation by fluorescence and images were captured using an Olympus BX-51 microscope equipped with a digital camera. For neural index measurements, TuJ1-labeled images and their corresponding DAPI images were opened in MetaMorph. Cell number was counted using the "count nuclei" plug-in, and manual adjustment was made to correct for any miscounted cells. To measure the area covered with neuritis, images were thresholded and the area covered by neurites was measured using region statistics. Neural index is expressed as neurite area/cell $\left(\mu \mathrm{m}^{2} /\right.$ cell $)$. Measurements represent a minimum of 2,000 cells from 5 images in 3 independent experiments for a total of at least 6,000 cells.

\section{RNA isolation and real-time polymerase chain reaction}

Total RNA was extracted from hNSCs cultured in the presence or absence of IGF-I using an RNeasy Kit (Qiagen) according to the manufacturers' instructions. Reverse

Table 1. Real-Time Polymerase Chain Reaction Primer Sequences

\begin{tabular}{lll}
\hline Target & \multicolumn{1}{c}{ Forward } & \multicolumn{1}{c}{ Reverse } \\
\hline IGF-I & CAACAAGCCCACAGGGTATGG & GCACTCCCTCTACTTGCGTTC \\
VEGF & CCATGGCAGAAGGAGGAGG & ATTGGATGGCACTAGCTGCG \\
GDNF & CTGACTTGGGTCTGGGCTATG & TTGTCACTCACCAGCCTTCTATT \\
BDNF & CCAAGGCAGGTTCAAGAGG & TCCAGCAGAAAGAGAAGAGGA \\
IGF-IR & CAATAAGTTCGTCCACAGAGACC & CCTCCTTTCCGGTAATAGTCTGT \\
TuJ1 & ATGCGGGAGATCGTGCACAT & CCCTGAGCGGACACTGT \\
GluR2 & TGTTGGAGTCCACGATGAACG & GCAAGATTTACTGGGGTTCCTAA \\
ChAT & CTGAATGACATGTATCTCAACAACC & TGTAGCTGAGTACACCAGAGATGAG \\
Syn & ACAAGACCGAGAGTGACCTCA & CGAGGAGTAGTCCCCAACTAAG \\
Actin & GCCGAGGACTTTGATTGC & GTGTGGACTTGGGAGAGG \\
\hline
\end{tabular}

BDNF, brain-derived neurotrophic factor; GDNF, glial-cell-derived neurotrophic factor; IGF-1, insulin-like growth factor-I; IGF-IR, type I IGF receptor; Syn, synaptophysin; VEGF, vascular endothelial growth factor. 
transcription was performed using the iScript cDNA Synthesis Kit (Bio-Rad). Real-time polymerase chain reactions (PCRs) were carried out in 96-well $0.2 \mathrm{~mL}$ PCR plates sealed with iCycler Optical Sealing tapes (Bio-Rad). PCR primers are listed in Table 1. Growth factor primers were previously described [1]. The PCR amplification profile was as follows: $95^{\circ} \mathrm{C}$ for $5 \mathrm{~min}, 40$ cycles of denaturation at $95^{\circ} \mathrm{C}$ for $30 \mathrm{~s}$, annealing at $58^{\circ} \mathrm{C}$ for $1 \mathrm{~min}$, and extension at $72^{\circ} \mathrm{C}$ for $30 \mathrm{~s}$, and a final phase of $72^{\circ} \mathrm{C}$ for $5 \mathrm{~min}$. The fluorescence threshold $C_{t}$ value was calculated by iCycler iQ system software and the levels were first normalized to the endogenous reference actin $\left(\Delta C_{t}\right)$, followed by relation to the control $\left(\Delta \Delta C_{t}\right)$ and expressed as $2^{-\Delta \Delta C t}$. PCR product levels were expressed as mean \pm SEM and a two-sample equal variance t-test was performed $(N=3$ replicates over 3 independent experiments).

\section{Western blotting}

Western blotting was performed as previously described [30]. hNSC lysates were prepared by lysing cells in modified RIPA buffer [20 mM Tris ( $\mathrm{pH} 7.4$ ), $150 \mathrm{mM}$ sodium chloride, $1 \mathrm{mM}$ ethylenediaminetetraacetic acid, $0.1 \%$ sodium dodecyl sulfate, $1 \mathrm{mM}$ sodium deoxycholate, 1\% Triton X-100, 0.1 trypsin units/L aprotinin, $10 \mathrm{mg} / \mathrm{mL}$ leupeptin, and $50 \mathrm{mg} / \mathrm{mL}$ phenylmethylsulfonyl fluoride (PMSF)]. Equal amounts of protein were loaded in each lane of a polyacrylamide gel, with gel percentages $(7.5 \%, 12.5 \%$, or $15 \%)$ dependent on the size of the protein of interest. Polyvinylidene fluoride membranes were incubated with primary antibody overnight at $4{ }^{\circ} \mathrm{C}$ and incubated with appropriate horseradish-peroxidase-conjugated secondary antibodies (Santa Cruz Biotechnology) for $1 \mathrm{~h}$ at $22^{\circ} \mathrm{C}$. Primary antibodies used are from Cell Signaling unless otherwise indicated: phosphop44/42 (Thr202/Tyr204) MAPK; phospho-Akt (Ser473); phospho-IGF-IR $\beta$ (Tyr1135/1136); p-mTOR (Ser2448); glyceraldehyde 3-phophate dehydrogenase (GAPDH; Chemicon). Antibody binding was developed with LumiGLO Reagent and Peroxide (Cell Signaling) and exposed to Kodak BioMax XAR film (Sigma). Each experimental paradigm was tested on 3 separate occasions using different cultures or tissue samples. Membranes were re-probed for GAPDH to confirm equal protein loading.

\section{TUNEL analysis}

hNSCs were grown and treated on glass coverslips followed by fixation in $4 \%$ PFA for $5 \mathrm{~min}$. TUNEL was used to detect DNA fragmentation as an indication of apoptotic death. hNSC plating densities were equal between treatment conditions. TUNEL analysis included blinded counting of at least 10 representative fields per condition in at least 3 independent experiments per our published protocol for an average total of $\sim 1,000-2,000$ hNSCs per condition. Samples were labeled with digoxygenin-dUTP and then stained with horseradish-peroxidase-conjugated anti-digoxygenin antibody using the ApopTag Plus In Situ Apoptosis Peroxidase Detection Kit (Chemicon). Alternatively, fluorescent TUNEL processing was carried out as described above with a fluorescein-labeled conjugate using the ApopTag Plus In Situ Apoptosis Fluorescein Detection Kit (Chemicon). Fluorescent signal was detected and recorded using an Olympus BX-51 microscope.

\section{Results}

\section{hNSC differentiation and growth factor production}

Previous studies documented the differentiation pattern and growth factor profile of hNSCs at extended differentiation time points [1]. Our goal was to examine hNSCs over a time period when they were plastic, that is, leaving their proliferative stage and entering terminal differentiation, a period when production and influence of endogenous growth factors were likely to be the most profound. mRNA was collected for quantitative real-time PCR from hNSCs that were either undifferentiated (UD) or had differentiated for either 3 or 7 days. Differentiation was initiated by removal of FGF, the sole mitogen required to maintain hNSC proliferation, from the medium. UD hNSCs were grown for $24 \mathrm{~h}$ before mRNA collection. Day 3 (D3) and day 7 (D7) hNSCs were cultured in the differentiation medium containing no FGF. UD hNSCs exhibited baseline levels of IGF-I, VEGF, BDNF, or GDNF (Fig. 1A). At D3, an increase in the expression level of both IGF-I and VEGF was detected (Fig. $1 B)$. By D7, there was a 2.5 -fold increase in IGF-I gene expression with continued low-level expression of VEGF (Fig. 1C). Baseline levels of IGF-IR were detectable in UD hNSCs, and increased at both D3 and D7 (Fig. 1D). These data demonstrate that IGF-I and VEGF are endogenously produced during early differentiation.

We next examined whether the addition of IGF-I during the early differentiation process alters neurotransmitters or maturation markers in hNSCs using quantitative real-time PCR. Over the course of 7 days of differentiation, we observed a temporally appropriate increase in TuJ1 (an early marker of neurons) and Syn (a synaptic marker) compared to UD cells (Fig. 1E, F). We also observed a 48-fold increase in GluR2 expression. After the addition of IGF-I, we did not detect significant changes in gene expression for any of the examined genes (Fig. 1D-H). Likewise, there was no change on growth factor production (data not shown). These data demonstrate that additional IGF-I has no effect on the expression profile during hNSC differentiation.

To confirm the identity and maturation of hNSCs into neurons at D7, we next investigated protein localization by ICC labeling. We observed expression of IGF-IR along the cell surface and neurites of hNSCs at D7 (Fig. 1I). The striking increase in expression of GluR2 at D7 was reflected by ICC and we detected extensive localization on the cell body and along neurites (Fig. 1J). Two neurotransmitter markers of cell fate expressed by hNSCs, VGlut2, and GAD [1] were highly expressed in all hNSCs at D7 (Fig. 1K, L). At D7 we observed no expression of astrocyte or oligodendrocyte markers (GFAP and Olig2, respectively; data not shown). Finally, we examined the localization of Syn along neurites in culture. Syn-positive terminals were detected on a small subset of neurites at D7 indicative of synapse formation (Fig. 1M, N). These data support the extensive neural differentiation and maturation of hNSCs by D7 of culture.

\section{IGF-I effects on neurite outgrowth and proliferation}

IGF-I promotes axonal outgrowth [29,31-33]; therefore, we next assessed the effect of IGF-I on hNSC neurite outgrowth as an early indicator of NSC neuronal differentiation [34]. We 
FIG. 1. Growth factor and differentiation makers were measured by polymerase chain reaction analysis of hNSCs over the first 7 days of differentiation. (A-C) Expression of IGF-I, VEGF, BDNF, and GDNF. Comparison between control hNSC and hNSC supplemented with $10 \mathrm{nM}$ IGF-I after 7 days of differentiation examining (D) IGF-IR, (E) TuJ1, (F) Syn, (G) GluR2, and (H) ChAT. Gene expression is first normalized to actin and then results are presented as fold changes against UD samples. Protein distribution in D7 hNSC for (I) IGFIR, (J) GluR2, (K) VGlut2, and (L) GAD. (M, N) Syn-positive terminals labeling synaptic terminals compared without or with IGF-I, respectively. Scale bar $=20 \mu \mathrm{m}$. BDNF, brainderived neurotrophic factor; GDNF, glial-cell-derived neurotrophic factor; hNSCs, human neural stem cells; IGF-1, insulin-like growth factor-I; IGF-IR, type I IGF receptor; UD, undifferentiated; Syn, synaptophysin; VEGF, vascular endothelial growth factor. Color images available online at www.liebertonline.com/scd.

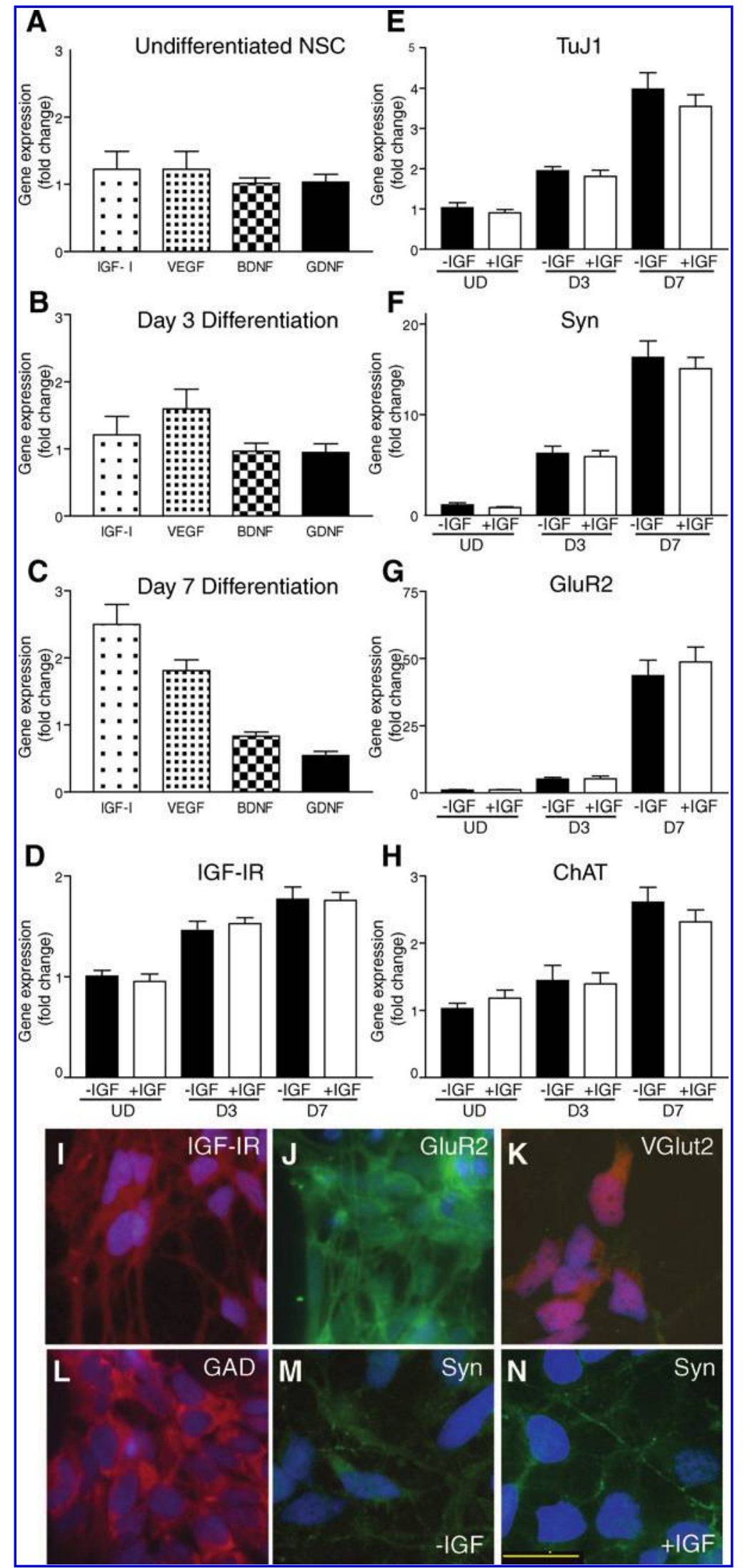


cultured hNSCs as a monolayer on glass coverslips in the presence or absence of IGF-I for the first 7 days of differentiation. Both the number of hNSCs with neurites and neurite length were quantified for UD hNSCs after $24 \mathrm{~h}$ of IGF-I, and D3 hNSCs cultured for $72 \mathrm{~h}$ in IGF-I. hNSCs were immunolabeled with NF to observe primary neuronal processes, and 500-700 individual cells were counted per condition over 3 experiments. Neurites were assessed as projections from the cell body $\geq 2$ cell diameters. Under UD hNSC conditions after $24 \mathrm{~h}$ of IGF-I treatment, we detected an increase in the number of hNSCs with neurites; however, this did not reach statistical significance. By D3, IGF-I treatment resulted in $\sim 20 \%$ of hNSCs extending primary neurites compared to only $5 \%$ of untreated hNSCs (Fig. 2A). We next measured neurite length using ImageJ [35]. Neurite length of UD hNSCs was not significantly increased by IGF-I treatment. At D3, IGF-I-induced neurite growth was significantly increased from 191 to $233 \mathrm{~nm}(P<0.01$; Fig. 2B). By D7, hNSCs require a high plating density for survival precluding the low culture density conditions necessary for observing single neurites. To examine the effects of IGF-I at D7, we relied on image analysis to measure a neural index. The neural index is expressed as the complete neuronal area divided by the number of nuclei, and results are presented as neurite area per cell $\left(\mu \mathrm{m}^{2} /\right.$ cell). hNSCs were immunolabeled by TuJ1 (neurites) and stained with DAPI (nuclei), which allowed us to take into account any variability in density across experiments. For validation, 6 images per condition were counted from 3 individual experiments representing over 6,000 DAPI-labeled cells. DAPI-positive cells were counted using MetaMorph image analysis software, and the corresponding TuJ1-positive image was inclusively thresholded to measure the total area covered in TuJ1-positive neurites (Fig. 2C). This method does not measure pixel intensity, only the area covered in neurites. Using this method, D3 samples treated with IGF-I demonstrated a significant increase in the neural index compared to untreated cells $\left(10 \mu \mathrm{m}^{2} /\right.$ cell vs. $4.7 \mu \mathrm{m}^{2} /$ cell, respectively; $P<0.001$; Fig. $\left.2 \mathrm{~F}\right)$. The D3 data confirmed that this method was sensitive enough to detect small changes in neurite outgrowth. At D7, IGF-I treatment for the duration of culture significantly increased the neural index from 70 to $91 \mu \mathrm{m}^{2} /$ cell $(P<0.02$; Fig. 2I), indicating a sustained effect on neurite length at D7. These data demonstrate that IGF-I stimulates hNSC neurite outgrowth and enhances NSC maturation over the first 7 days of differentiation.

Neurite outgrowth normally occurs in postmitotic neurons. We next examined the effects of IGF-I on cell proliferation. hNSC cultures at UD, D3, or D7 were incubated with EdU for $2 \mathrm{~h}$ before fixation. Approximately 1,000 cells were counted per experiment for the UD samples $(N=3)$. For D3 and D7 hNSCs, which are plated at higher density, a minimum of 3,000 cells were counted per experiment $(N=3$; Fig. 3B). Approximately $27 \%$ of untreated UD hNSCs were EdU positive (Fig. 3A; Control UD). When UD hNSCs were treated with IGF-I, we detected a small reduction in EdU incorporation (25\%; Fig. 3A). At D3, the effect of IGF-I treatment was significant with a reduction in EdU-positive nuclei from $17 \%$ to $14 \%(P<0.02$; Fig. 3A). We did not observe any change at D7, suggesting that the effects on proliferation are only associated with the earlier time points (Fig. $3 \mathrm{~A})$. These data demonstrate that treatment of hNSC cultures with IGF-I does not promote or maintain proliferation during initial stages of differentiation.

\section{IGF-I-mediated signaling}

IGF-I signaling stimulates neurite outgrowth via either AKT or MAPK $[8,29,33,36-38]$; therefore, we investigated which signaling mechanisms are active in hNSCs and responsible for IGF-I-mediated neurite outgrowth. hNSCs were treated with IGF-I and the activation was examined for up to $2 \mathrm{~h}$ to examine the time course of down stream signaling. The specificity of IGF-I signaling was assessed using pathway-specific inhibitors of MAPK (U0126; U) or AKT (LY294002; LY), or with the IGF-IR inhibitor NVP-AEW541 (NVP). For all hNSCs (UD, D3, and D7), we detected IGF-I activation of IGF-IR within 5 min of stimulation (Fig. 4A-C). Levels of IGF-IR were considerably lower in UD samples, requiring additional amplification with a stronger enhanced chemiluminescence (ECL) reagent to observe receptor phosphorylation (Fig. 4A). With amplification, we did observe the same pattern of IGF-IR activation at D3 and D7. IGF-IR signaling was abolished in the presence of IGF-IR antagonist, NVP. In UD hNSCs, we observed low levels of basal AKT activity and rapid activation of AKT signaling within $5 \mathrm{~min}$ of stimulation. AKT activation returned to near baseline $2 \mathrm{~h}$ after stimulation. The same pattern was observed at D3 and D7 (Fig. 4B, C). We next examined mTOR, a downstream effector of AKT signaling associated with enhancing NSC differentiation [39]. We detected little mTOR phosphorylation in UD hNSC samples (Fig. 4A). At D3 and D7, we observe phosphorylation of mTOR in response to IGF-I signaling (Fig. 4B, C). In UD hNSCs, MAPK signaling was activated by $5 \mathrm{~min}$ and maintained for up to $30 \mathrm{~min}$. Under basal conditions, we detected low levels of MAPK signaling at D3 and D7. After IGF-I stimulation, there was a robust increase in MAPK signaling by $5 \mathrm{~min}$, which was maintained over the course of the experiment. hNSCs treated with NVP and IGF-I or NVP alone at D7 exhibited a reduction in MAPK activation below control conditions. When MAPK signaling was inhibited we observed increased AKT phosphorylation, suggesting that inhibition of IGF-I-mediated MAPK signaling is compensated for by AKT. The reverse, however, does not occur, and attenuation of AKT signaling did not result in increased MAPK activation (Fig. 4A-C). Inhibitor-mediated changes in IGF-I signaling were maintained under all experimental conditions (Fig. 4A-C).

We then investigated which downstream signaling pathway was required for IGF-I-mediated neurite outgrowth. hNSCs were treated with IGF-I with or without inhibitors for $24 \mathrm{~h}$ before fixation and ICC for NF to assess primary neurite outgrowth. By $24 \mathrm{~h}$, IGF-I promotes increased neurite outgrowth compared to the control that remain rounded (Fig. 4D, E). When IGF-I signaling was inhibited with either NVP or LY, IGF-I-induced neurite outgrowth was lost and the hNSCs maintained their round UD morphology (Fig. 4F, G). When MAPK signaling was inhibited with U, IGF-I-mediated neurite outgrowth was maintained (Fig. $4 \mathrm{H}$ ). Neurite growth in the presence of the inhibitors for 3 or 7 days resulted in increased cell death, suggesting that over a longer period both MAPK and AKT are required for survival and growth. Together these data demonstrate that IGF-I-induced neurite outgrowth is mediated via AKT but not MAPK in this system. 

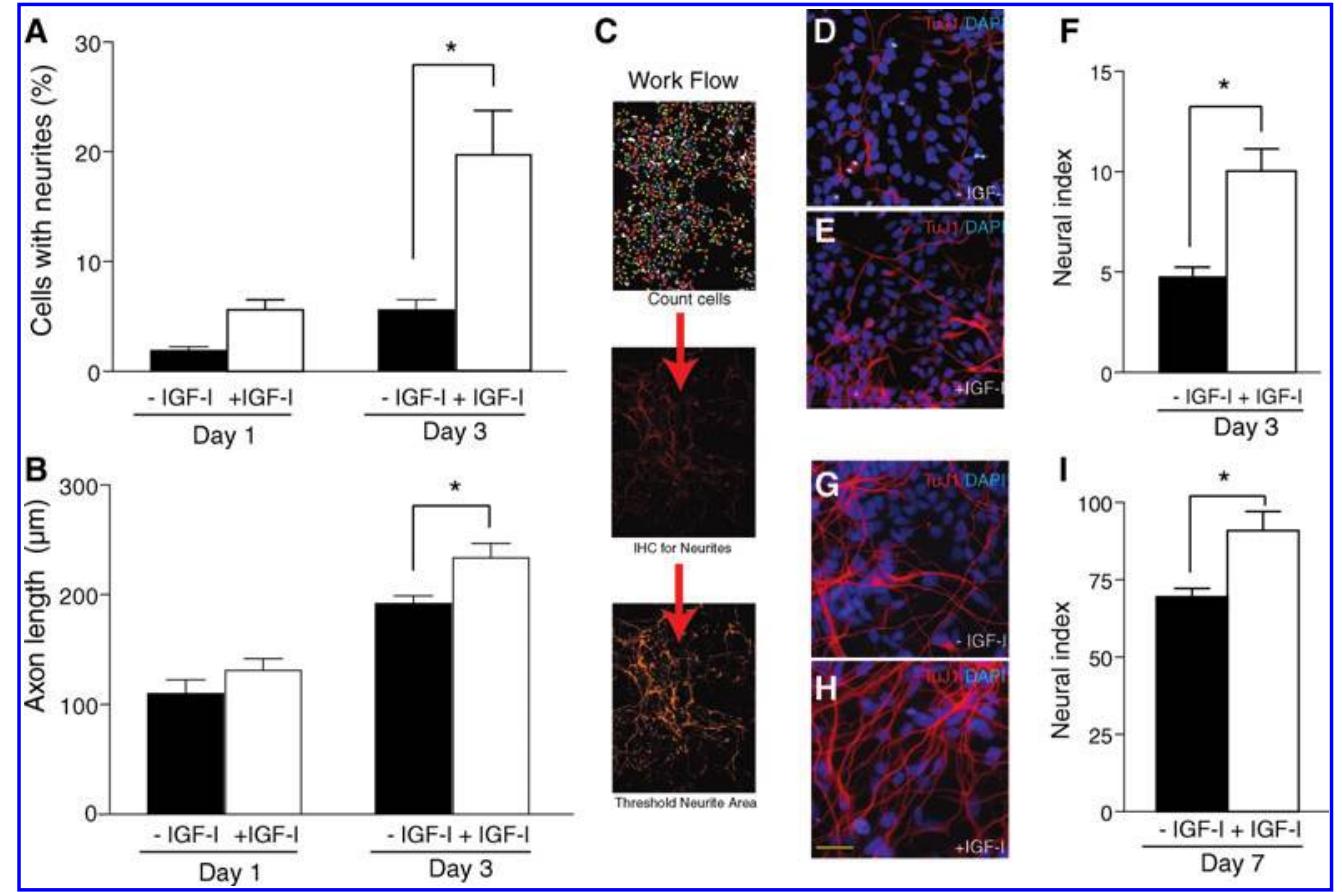

FIG. 2. IGF-I increases neurite outgrowth in differentiating hNSC. (A) An increase in number of neurites is observed in hNSC cultures with or without IGF-I in either UD or D3 cells. (B) Individual neurite length increases with IGF-I in either UD or D3 cells. (C) Illustration of the work flow for calculating the neural index. (D, E, G, H) Representative images demonstrating the extent of TuJ1

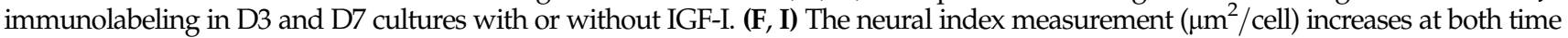
points after IGF-I treatment compared to the untreated control. ${ }^{*} P<0.05$. Scale bar $=50 \mu \mathrm{m}$. D3, day 3; D7, day 7; hNSCs, human neural stem cells; IGF-1, insulin-like growth factor-I; UD, undifferentiated. Color images available online at www.liebertonline.com/scd.

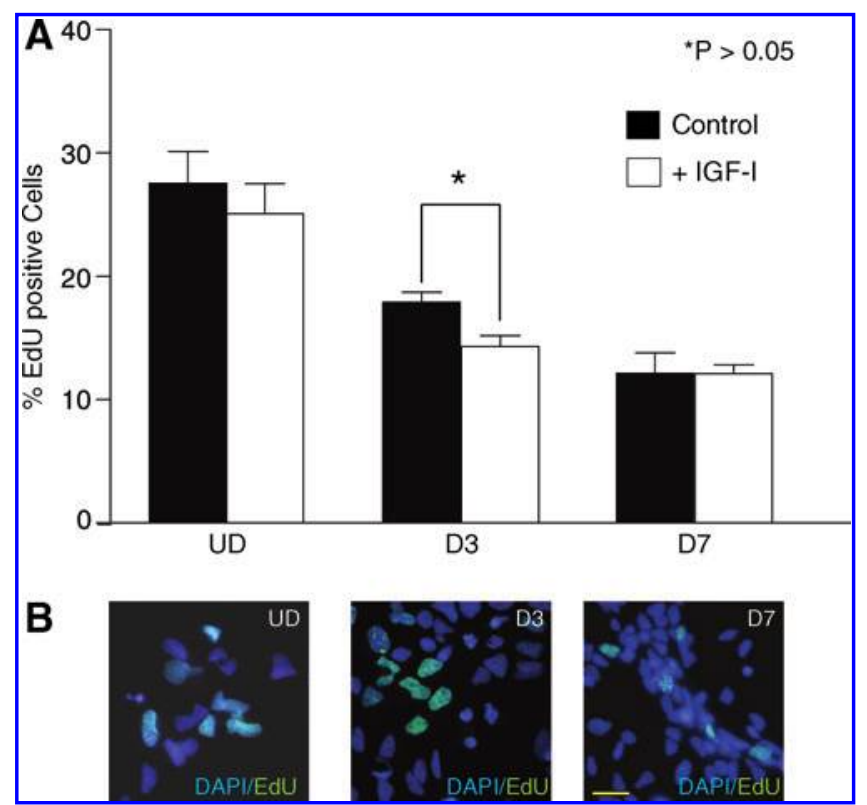

FIG. 3. Effects of IGF-I on hNSC proliferation. (A) The percentage of EdU-positive cells for UD, D3, and D7 hNSC with and without IGF-I. (B) Representative image of each timepoint-labeled DAPI (blue) and EdU (green); note fewer EdUlabeled cells over time. Scale bar $=50 \mu \mathrm{m}$. D3, day 3; D7, day 7; EdU, 5'-ethynyl-2'-deoxyuridine; hNSCs, human neural stem cells; IGF-1, insulin-like growth factor-I; UD, undifferentiated. Color images available online at www.liebertonline.com/scd.

\section{IGF-I-mediated neuroprotection of hNSCs}

Glutamate excitotoxicity is one proposed pathophysiological mechanism for neurodegeneration in a variety of diseases such as stroke and ALS; therefore, we examined the effects of excitotoxicity on hNSC and the neuroprotective potential of IGF-I [40]. We first assessed the susceptibility of D7 hNSCs to glutamate excitotoxicity by treating hNSCs with increasing concentrations of glutamate from 1 to $200 \mu \mathrm{M}$. The hNSCs were fixed and cell death measured by assessing DNA fragmentation via TUNEL labeling. TUNEL-positive cells were counted and expressed as a percentage of the total cells. With increasing concentrations of glutamate, there was a dose-dependent increase in cell death (Fig. 5A). Maximal cell death was observed at $100 \mu \mathrm{M}$ glutamate, which resulted in $\sim 28 \%$ cell death. To investigate the ability of IGF-I to protect against glutamate-induced cell death, D7 hNSCs were cocultured with $100 \mu \mathrm{M}$ glutamate and $10 \mathrm{nM}$ IGF-I. After $24 \mathrm{~h}$, hNSCs were fixed and assessed for cell death. IGF-I reduced the level of hNSC death back to baseline in the presence of $100 \mu \mathrm{M}$ glutamate $(P<0.001$; Fig. 5B). These data suggest that IGF-I is protective against glutamate excitotoxic stress in neurons differentiated from hNSCs.

\section{Discussion}

hNSCs in combination with IGF-I may be a more potent therapy than either alone for the treatment of both neurodegenerative diseases and neuronal injuries. In this study we 


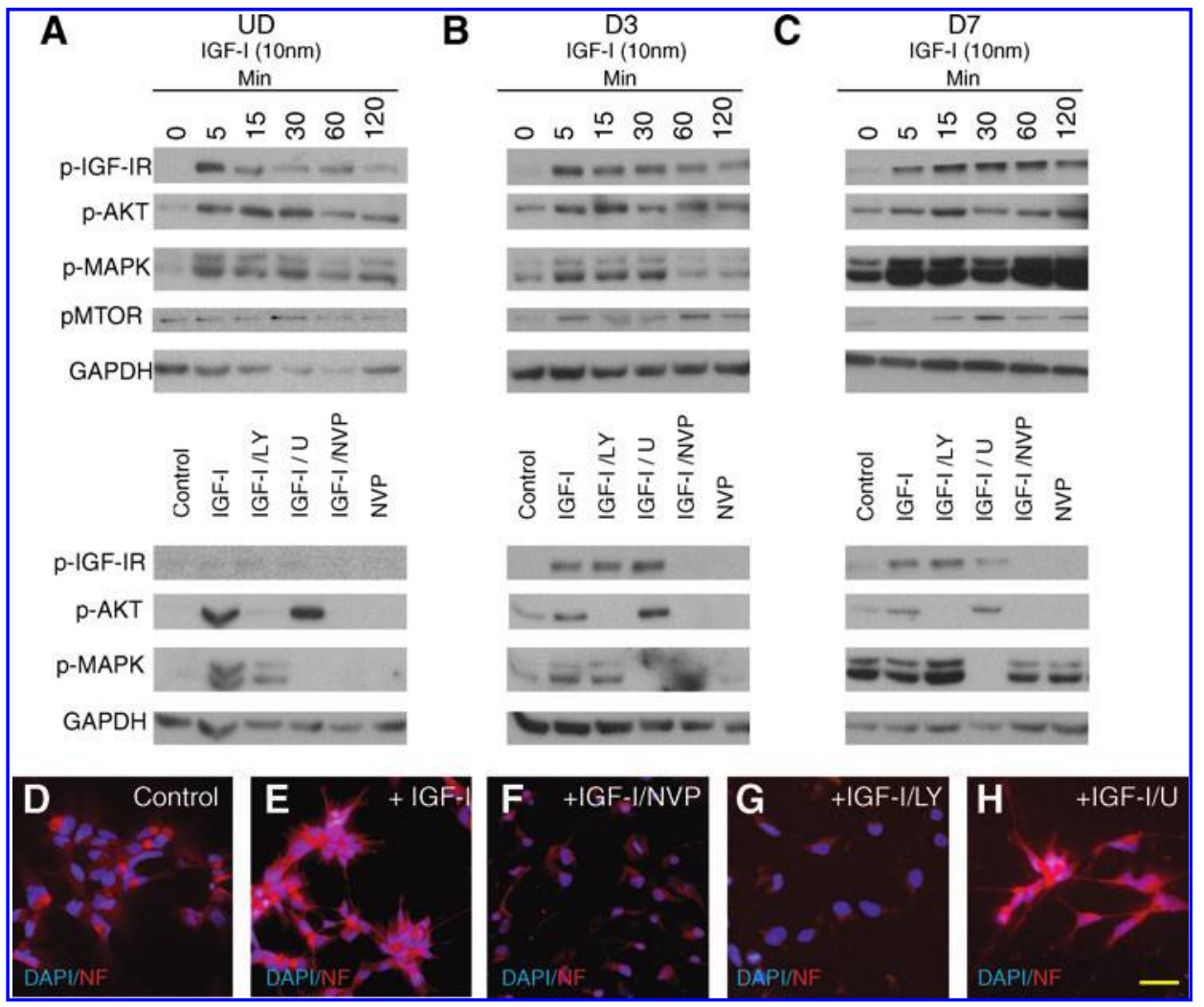

FIG. 4. IGF-I-mediated signaling in hNSC. Western blot analysis of IGF-I signaling of UD, D3, and D7 hNSC. hNSC treated with either a time course of IGF-I $(10 \mathrm{nM})$ stimulation between 0 and $120 \mathrm{~min}$, or an inhibitor panel of LY, U, or NVP followed by $30 \mathrm{~min}$ IGF-I stimulation. (A) UD hNSC, (B) D3, and (C) D7 hNSC. All westerns were probed with pIGF-IR, pAKT, pMAPK, and pmTOR. GAPDH was used as a loading control. (D-H) Effect of signaling inhibition on IGF-Imediated axon outgrowth of hNSC. Immunocytochemistry labeling of neurofilament indicating primary neurite formation. Culture conditions: (D) control, (E) IGF-I alone, (F) IGFI+NVP, (G) IGF-I+LY, and (H) IGF-I+U. All inhibitors used at $10 \mu \mathrm{M}$ concentration. Scale bar hairsp; $=50 \mu \mathrm{m}$. D3, day 3; D7, day 7; GAPDH, glyceraldehyde 3-phophate dehydrogenase; hNSCs, human neural stem cells; IGF-1, insulin-like growth factor-I; IGF-IR, type I IGF receptor; UD, undifferentiated. Color images available online at www.liebertonline.com/scd.

demonstrated the positive effects of IGF-I on hNSC differentiation. We report that IGF-I increases neurite outgrowth, decreases proliferation, and is neuroprotective against glutamate excitotoxicity. Our findings demonstrate for the first time that IGF-I has a beneficial effect on the differentiation and survival of hNSCs and a proliferative effect on UD hNSCs.
In this study we focus on the initial 7 days of differentiation as we speculate that this is a critical period when $\mathrm{hNSCs}$ are plastic, that is, leaving the proliferative phase of their development and beginning terminal differentiation. hNSCs are directed along differentiation pathways by extracellular signaling molecules, including IGF-I [39,41,42]. We examined expression of 4 growth factors by PCR: GDNF, BDNF, VEGF,

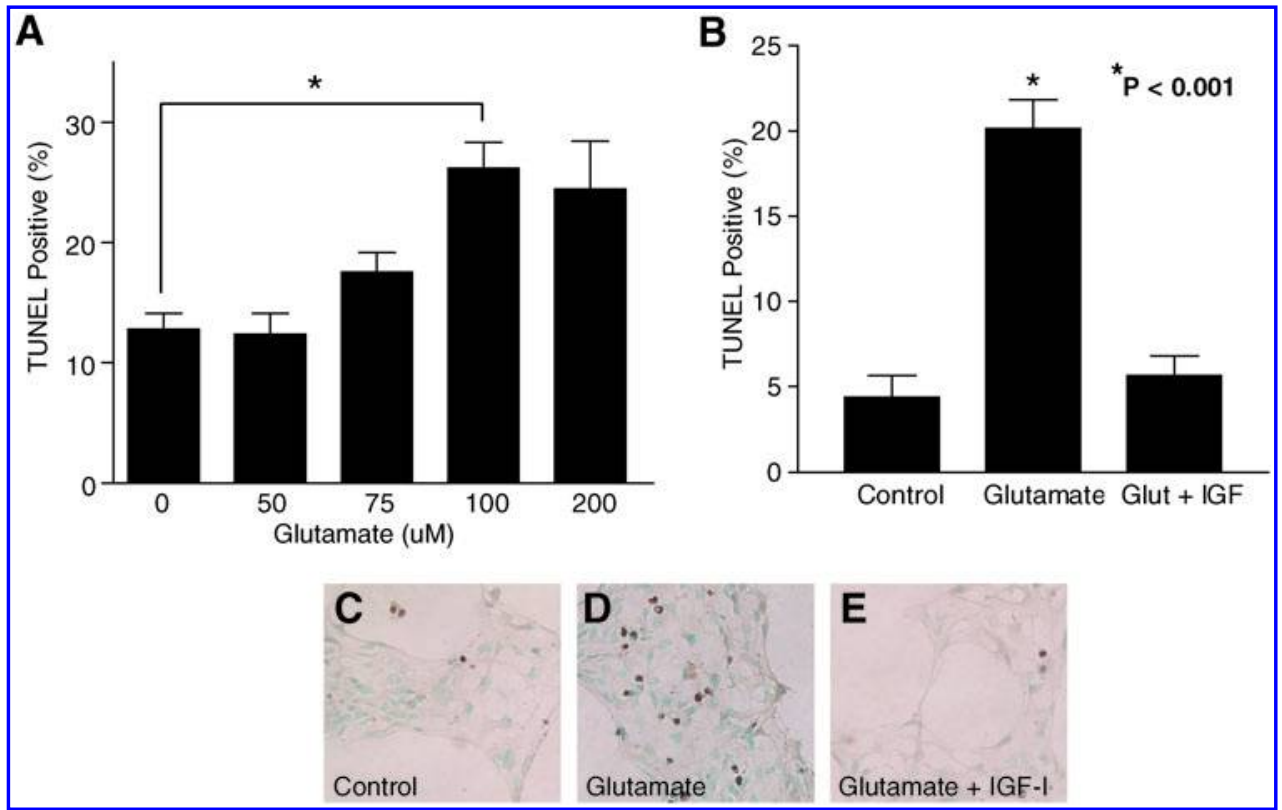

FIG. 5. IGF-I-mediated neuroprotection of hNSC. Cell death after glutamate exposure was assessed by DNA fragmentation (TUNEL). (A) Glutamate kill curve: increasing concentrations of glutamate from 50 to $200 \mu \mathrm{M}$ for $24 \mathrm{~h}$ increased levels of cell death up to $100 \mu \mathrm{M}\left({ }^{*} P<0.01\right)$. (B) IGF-I prevents glutamate-induced cell death compared to control $\left({ }^{*} \mathrm{P}<0.001\right)$. (C-E) Representative images of TUNELpositive hNSC. hNSCs, human neural stem cells; IGF-1, insulin-like growth factor-I. Color images available online at www.liebertonline.com/scd. 
and IGF-I (Fig. 1). UD hNSCs display baseline expression of all 4 of these growth factors. On D3 we observe an increase in IGF-I and VEGF expression. By D7, there are higher levels of IGF-I than VEGF. These findings are consistent with previous studies demonstrating that by D14 of differentiation, IGF-I is the most highly represented of these 4 growth factors [1]. In UD hNSCs, we detect low levels of IGF-IR expression by PCR (Fig. 1). Given the changes at the gene expression level do not necessarily reflect changes in protein expression or functional signaling, we assessed expression and activation of IGF-IR. Western blotting reveals phosphorylation of both AKT and MAPK (Fig. 4), indicating functional receptor expression and activation of IGF-IR signaling pathways. At D3 and D7, differentiating hNSCs exhibit consistent activation of MAPK and AKT in response to IGF-I stimulation. Incubation of hNSCs with the IGF-IR blocker NVP abolished IGF-I activation of both pathways at all time points. Interestingly, incubation with NVP alone at D7 without the addition of IGF-I also reduced MAPK below baseline, suggesting endogenous IGF-I autocrine stimulation of MAPK signaling (Fig. 4). VEGF is expressed by hNSCs at D3 and D7 and we have previously shown that VEGF activates MAPK signaling in primary neurons [43]. Endogenous IGF-I and VEGF production and signaling may account for the basal MAPK activation at these time points (Fig. 1). Together these findings demonstrate that hNSCs are an early source of endogenous growth factors and that they respond to IGF-I stimulation, with activation of both AKT and MAPK signaling.

We next examined if IGF-I treatment directed early differentiation of hNSCs. We detected a temporal increase in expression of several markers associated with neuronal differentiation and maturation, including the neuronal markers neuron-specific tubulin (TuJ1) and Syn (Fig. 1). Surprisingly, we did not observe any changes at the transcriptional level of either of these markers after IGF-I treatment, suggesting that IGF-I does not affect neuronal fate or rate of maturation per se. By D7, we did observe Syn-positive synaptic terminals along neurites, indicating that hNSCs are beginning to mature and form synaptic connections. PCR results indicate that, as hNSCs differentiate, there is an increasing level of Syn. By ICC we also observe that D7 hNSCs are interconnected by Syn-positive terminals (Fig. 1). These data demonstrate that by D7, hNSCs are maturing and creating neural networks; however, IGF-I does not affect differentiation at a transcriptional level of the markers we assessed.

Neurite outgrowth is one of the earliest indicators of postmitotic hNSCs becoming terminally differentiated $[34,44]$. We demonstrate that IGF-I increases the number and length of neurites in hNSCs by D3 of differentiation and IGFI-mediated axon outgrowth is maintained at D7 using a measure of neural index (Fig. 2). Using other cell types, we and others have shown that IGF-I directly impacts cytoskeletal rearrangement and enhances axon outgrowth $[20,32,45,46]$. Using signaling pathway-specific inhibitors, we observe that the IGF-I-mediated neurite outgrowth is mediated by AKT signaling (Fig. 4). These findings are consistent with IGF-I-mediated outgrowth in neuroblastoma cells, corticospinal neurons, and hippocampal neurons $[20,33,46]$. As hNSCs already express IGF-I, it may be possible that endogenous levels are insufficient to elicit maximal outgrowth. We do not detect changes in expression of different neural markers and suggest that IGF-I is enhancing and maintaining the development of hNSCs rather than directing differentiation. We anticipated a dual role for both AKT and MAPK signaling for neurite outgrowth based on previous studies on IGF-I action in both neuroblastoma and corticospinal neurons, hypothesizing that a common mechanism for signaling via MAPK would be required for outgrowth $[20,32,46]$. In these experiments, however, MAPK was not required for neurite outgrowth. mTOR mediates IGF-I-directed differentiation of forebrain-derived neural progenitor cells in vitro [39]. Consistent with these findings, we detected low levels of mTOR activation in UD hNSCs, while at later time points, especially at D7, we observed a robust activation of mTOR in response to IGF-I (Fig. 4). Together these findings highlight AKT signaling as the mechanism for IGF-Imediated neurite outgrowth in hNSCs.

On the basis of these findings demonstrating the positive effects of IGF-I on neurite outgrowth, we hypothesized that IGF-I may enhance neural development of hNSCs. IGF-I is known to promote proliferation downstream of both AKT and MAPK signaling $[47,48]$. We wanted to examine whether IGF-I maintained proliferation of hNSCs. One limitation of cellular therapies based from stem cell technology is the potential for aberrant proliferation and formation of teratomas [49]. We observe that across all time points IGF-I does not maintain or promote proliferation of hNSCs (Fig. 3). Both of these findings regarding neurite outgrowth and differentiation at the expense of proliferation are supported by results in hNSCs of oligodendrocyte fate, where IGF-I increases the proportion of hNSCs differentiating concurrent with a decrease in proliferation [42]. These results demonstrate that combining IGF-I with hNSCs as a combined therapy would not promote proliferation and lead to unwanted propagation.

The capacity of IGF-I to prevent programmed cell death has been demonstrated in many neural cell types under a variety of conditions, including $\mathrm{MNs}$ and sensory neurons $[10,11,15,17,50-55]$. One mechanism associated with $\mathrm{MN}$ cell death is via glutamate excitotoxicity [40,56,57]. We exposed hNSCs to glutamate to assess if hNSCs are susceptible to excitotoxic stress, and if so, is IGF-I neuroprotective. We observed $27 \%$ cell death after treatment with glutamate (Fig. 5). This is in contrast to primary MN that, under the same conditions, display up to $60 \%$ cell death [40]. These findings suggest that at D7, hNSCs are resistant to glutamate-induced cell death potentially due to an early neuronal differentiation state. Further differentiation of hNSCs, however, may confer increased sensitivity and therefore make them more susceptible to glutamate-induced cell death. The high levels of GluR2 expression (Fig. 1) may contribute to the increased excitotoxic resistance of hNSCs, given that the GluR2 subunit of the $\alpha$-amino-3-hydroxy5-methyl-4-isoxazolepropionic acid (AMPA) receptor regulates susceptibility to excitotoxic stress $[56,58,59]$. Yan et al. observed production of IGF-I up to the first month of differentiation before the level started to decrease; therefore, IGF-I may be protective in vivo [1].

\section{Conclusions}

Our findings demonstrate that IGF-I is produced early in hNSC differentiation. Direct treatment with IGF-I does not 
change the expression profile of hNSCs as they are differentiating, suggesting that IGF-I does not affect lineage selection, but rather enhances neural development and neurite outgrowth. Signaling via AKT, but not MAPK, mediates IGFI-stimulated neurite outgrowth. hNSCs are more resistant to glutamate excitotoxicity than mature MNs. As such, hNSCs may be less susceptible to pathogenic factors while they mature; however, IGF-I remains a potent neuroprotective factor for excitotoxic stress in hNSCs. Finally, IGF-I does not promote proliferation of hNSCs. Our data support the idea that upregulation of IGF-I production in hNSCs may offer additional therapeutic benefits when hNSCs engineered to overexpress IGF-I are transplanted into the nervous system of animal models and humans with neurological disorders.

\section{Acknowledgments}

This work was supported by the A. Alfred Taubman Medical Institute and the Program for Neurology Research and Discovery. The authors thank Drs. Stacey Sakowski and Kelli Sullivan for scientific input and editorial assistance.

\section{Author Disclosure Statement}

Dr. Karl Johe has a financial interest in Neuralstem, Inc. The remaining authors would like to state that they have no commercial associations that might create a conflict of interest in connection with this article.

\section{References}

1. Yan J, L Xu, AM Welsh, G Hatfield, T Hazel, K Johe and VE Koliatsos. (2007). Extensive neuronal differentiation of human neural stem cell grafts in adult rat spinal cord. PLoS Med 4:e39.

2. $\overline{\mathrm{Xu}} \mathrm{L}, \mathrm{DK}$ Ryugo, T Pongstaporn, K Johe and VE Koliatsos. (2009). Human neural stem cell grafts in the spinal cord of SOD1 transgenic rats: differentiation and structural integration into the segmental motor circuitry. I Comp Neurol 514:297-309.

3. Cizkova D, O Kakinohana, K Kucharova, S Marsala, K Johe, T Hazel, MP Hefferan and M Marsala. (2007). Functional recovery in rats with ischemic paraplegia after spinal grafting of human spinal stem cells. Neuroscience 147:546-560.

4. Xu L, J Yan, D Chen, AM Welsh, T Hazel, K Johe, G Hatfield and VE Koliatsos. (2006). Human neural stem cell grafts ameliorate motor neuron disease in SOD-1 transgenic rats. Transplantation 82:865-875.

5. Anlar B, KA Sullivan and EL Feldman. (1999). Insulin-like growth factor-I and central nervous system development. Horm Metab Res 31:120-125.

6. Russo VC, P Gluckman, EL Feldman and GA Werther. (2005). The insulin-like growth factor system and its pleiotropic functions in brain. Endocr Rev 26:916-943.

7. Ishii DN and SB Lupien. (1995). Insulin-like growth factors protect against diabetic neuropathy: effects on sensory nerve regeneration in rats. J Neurosci Res 40:138-144.

8. Cheng HL, M Steinway, CL Delaney, TF Franke and EL Feldman. (2000). IGF-I promotes Schwann cell motility and survival via activation of Akt. Mol Cell Endocrinol 170: 211-215.

9. Gandhi D, CC Matthews, EL Feldman, N Hotta, DA Greene, JD Ward, AAF Sima and AJM Boulton. (1995). IGF-I rescues neurons from hyperglycemic, hyperosmotic injury. In: Diabetic Neuropathy: New Concepts and Insights. Hotta N, DA Greene, JD Ward, AAF Sime and AJM Boulton, eds. Elsevier Science B.V., Amsterdam, pp 183-189.

10. Sullivan KA, B Kim, JW Russell, EL Feldman and E Muller. (1998). IGF-I in neuronal differentiation and neuroprotection. In: IGFs in the Nervous System. Müller EE, ed. SpringerVerlag Italia Srl, Milan, Italy, pp 28-46.

11. Vincent AM, C Consilvio and EL Feldman. (2003). IGF-Imediated protection of motor neurons in a cell culture model of ALS. Endocrine Society Abstracts P3-316:548.

12. Stewart CE and P Rotwein. (1996). Growth, differentiation, and survival: multiple physiological functions for insulinlike growth factors. Physiol Rev 76:1005-1026.

13. Pang Y, B Zheng, LW Fan, PG Rhodes and Z Cai. (2007). IGF-1 protects oligodendrocyte progenitors against TNFalpha-induced damage by activation of PI3K/Akt and interruption of the mitochondrial apoptotic pathway. Glia 55:1099-1107.

14. Zaka M, MA Rafi, HZ Rao, P Luzi and DA Wenger. (2005). Insulin-like growth factor-1 provides protection against psychosine-induced apoptosis in cultured mouse oligodendrocyte progenitor cells using primarily the PI3K/Akt pathway. Mol Cell Neurosci 30:398-407.

15. Ness JK, RC Scaduto, Jr. and TL Wood. (2004). IGF-I prevents glutamate-mediated bax translocation and cytochrome C release in O4+ oligodendrocyte progenitors. Glia 46:183-194.

16. Delaney CL, HL Cheng and EL Feldman. (1999). Insulin-like growth factor-I prevents caspase-mediated apoptosis in Schwann cells. J Neurobiol 41:540-548.

17. Hodge RD, AJ D'Ercole and JR O'Kusky. (2007). Insulin-like growth factor-I (IGF-I) inhibits neuronal apoptosis in the developing cerebral cortex in vivo. Int J Dev Neurosci 25:233-241.

18. Castrillo A, OG Bodelon and L Bosca. (2000). Inhibitory effect of IGF-I on type 2 nitric oxide synthase expression in Ins-1 cells and protection against activation-dependent apoptosis. Diabetes 49:209-217.

19. Vincent AM, EL Feldman, DK Song, V Jung, A Schild, W Zhang, MJ Imperiale and NM Boulis. (2004). Adeno-associated viral-mediated insulin-like growth factor delivery protects motor neurons in vitro. Neuromolecular Med 6:79-85.

20. Ozdinler PH and JD Macklis. (2006). IGF-I specifically enhances axon outgrowth of corticospinal motor neurons. Nat Neurosci 9:1371-1381.

21. Hollis ER, 2nd, P Lu, A Blesch and MH Tuszynski. (2009). IGF-I gene delivery promotes corticospinal neuronal survival but not regeneration after adult CNS injury. Exp Neurol 215:53-59.

22. Nayak MS, YS Kim, M Goldman, HS Keirstead and DA Kerr. (2006). Cellular therapies in motor neuron diseases. Biochim Biophys Acta 1762:1128-1138.

23. Lunn JS, MP Hefferan, M Marsala and EL Feldman. (2009). Stem cells: comprehensive treatments for amyotrophic lateral sclerosis in conjunction with growth factor delivery. Growth Factors 27:133-140.

24. Suzuki M and CN Svendsen. (2008). Combining growth factor and stem cell therapy for amyotrophic lateral sclerosis. Trends Neurosci 31:192-198.

25. Hedlund E, MP Hefferan, M Marsala and O Isacson. (2007). Cell therapy and stem cells in animal models of motor neuron disorders. Eur J Neurosci 26:1721-1737.

26. Suzuki M, J McHugh, C Tork, B Shelley, SM Klein, P Aebischer and CN Svendsen. (2007). GDNF secreting human 
neural progenitor cells protect dying motor neurons, but not their projection to muscle, in a rat model of familial ALS. PLoS ONE 2:e689.

27. Klein SM, S Behrstock, J McHugh, K Hoffmann, K Wallace, M Suzuki, P Aebischer and CN Svendsen. (2005). GDNF delivery using human neural progenitor cells in a rat model of ALS. Hum Gene Ther 16:509-521.

28. Johe KK, TG Hazel, T Muller, MM Dugich-Djordjevic and RD McKay. (1996). Single factors direct the differentiation of stem cells from the fetal and adult central nervous system. Genes Dev 10:3129-3140.

29. Kim B, PS Leventhal, AR Saltiel and EL Feldman. (1997). Insulin-like growth factor-I-mediated neurite outgrowth in vitro requires MAP kinase activation. J Biol Chem 272: 21268-21273.

30. Vincent AM, EL Feldman, DK Song, V Jung, A Schild, W Zhang, MJ Imperiale and NM Boulis. (2004). Adenoassociated viral-mediated insulin-like growth factor delivery protects motor neurons in vitro. Neuromolecular Med 6:79-86.

31. Leventhal PS, EA Shelden, B Kim and EL Feldman. (1997). Tyrosine phosphorylation of paxillin and focal adhesion kinase during insulin-like growth factor-I-stimulated lamellipodial advance. I Biol Chem 272:5214-5218.

32. Kim B, PS Leventhal, AR Saltiel and EL Feldman. (1997). Insulin-like growth factor-I-mediated neurite outgrowth in vitro requires mitogen-activated protein kinase activation. I Biol Chem 272:21268-21273.

33. Laurino L, XX Wang, BA de la Houssaye, L Sosa, S Dupraz, A Caceres, KH Pfenninger and S Quiroga. (2005). PI3K activation by IGF-1 is essential for the regulation of membrane expansion at the nerve growth cone. J Cell Sci 118:3653-3662.

34. Kim SJ, TG Son, K Kim, HR Park, MP Mattson and J Lee. (2007). Interferon-gamma promotes differentiation of neural progenitor cells via the JNK pathway. Neurochem Res 32: 1399-1406.

35. Abramoff MD, PJ Magelhaes and SJ Ram. (2004). Image processing with ImageJ. Biophotonics Int 11:36-42.

36. Kim B, PS Leventhal and EL Feldman. (1996). Insulin-like growth factor-I (IGF-I) induces localization of RAC and phosphatidylinositol 3-kinase (PI3-K) to membrane ruffles. Mol Biol Cell 7:342a.

37. Kim B and EL Feldman. (1998). Differential regulation of focal adhesion kinase and mitogen-activated protein kinase tyrosine phosphorylation during insulin-like growth factorI-mediated cytoskeletal reorganization. I Neurochem 71: 1333-1336.

38. Leventhal PS, EA Shelden, B Kim and EL Feldman. (1997). Tyrosine phosphorylation of paxillin and focal adhesion kinase during insulin-like growth factor-I-stimulated lamellipodial advance. J Biol Chem 272:5214-5218.

39. Han J, B Wang, Z Xiao, Y Gao, Y Zhao, J Zhang, B Chen, $X$ Wang and J Dai. (2008). Mammalian target of rapamycin (mTOR) is involved in the neuronal differentiation of neural progenitors induced by insulin. Mol Cell Neurosci 39: $118-124$.

40. Vincent AM, BC Mobley, A Hiller and EL Feldman. (2004). IGF-I prevents glutamate-induced motor neuron programmed cell death. Neurobiol Dis 16:407-416.

41. McCurdy RD, F Feron, JJ McGrath and A Mackay-Sim. (2005). Regulation of adult olfactory neurogenesis by insulin-like growth factor-I. Eur J Neurosci 22:1581-1588.

42. Hsieh J, JB Aimone, BK Kaspar, T Kuwabara, K Nakashima and FH Gage. (2004). IGF-I instructs multipotent adult neural progenitor cells to become oligodendrocytes. J Cell Biol 164:111-122.

43. Lunn JS, SA Sakowski, B Kim, AA Rosenberg and EL Feldman. (2009). Vascular endothelial growth factor prevents G93A-SOD1-induced motor neuron degeneration. Dev Neurobiol 69:871-884.

44. Ohuchi T, S Maruoka, A Sakudo and T Arai. (2002). Assaybased quantitative analysis of PC12 cell differentiation. I Neurosci Methods 118:1-8.

45. Recio PE, MM Rechler and DN Ishii. (1986). Effects of insulin, insulin-like growth factor-II, and nerve growth factor on neurite formation and survival in cultured sympathetic and sensory neurons. J Neurosci 6:1211-1219.

46. Kurihara S, F Hakuno and S Takahashi. (2000). Insulin-like growth factor-I-dependent signal transduction pathways leading to the induction of cell growth and differentiation of human neuroblastoma cell line SH-SY5Y: the roles of MAP kinase pathway and PI 3-kinase pathway. Endocr J 47: 739-751.

47. Blakesley VA, T Kalebic, LJ Helman, B Stannard, TN Faria, CT Roberts, Jr. and D LeRoith. (1996). Tumorigenic and mitogenic capacities are reduced in transfected fibroblasts expressing mutant insulin-like growth factor (IGF)-I receptors. The role of tyrosine residues 1250, 1251, and 1316 in the carboxy-terminus of the IGF-I receptor. Endocrinology 137:410-417.

48. Kalluri HS, R Vemuganti and RJ Dempsey. (2007). Mechanism of insulin-like growth factor I-mediated proliferation of adult neural progenitor cells: role of Akt. Eur J Neurosci 25:1041-1048.

49. Blum B and N Benvenisty. (2008). The tumorigenicity of human embryonic stem cells. Adv Cancer Res 100:133-158.

50. Leinninger GM, C Backus, MD Uhler, SI Lentz and EL Feldman. (2004). Phosphatidylinositol 3-kinase and Akt effectors mediate insulin-like growth factor-I neuroprotection in dorsal root ganglia neurons. FASEB J 18:1544-1546.

51. Brywe KG, C Mallard, M Gustavsson, M Hedtjarn, AL Leverin, $\mathrm{X}$ Wang, $\mathrm{K}$ Blomgren, $\mathrm{J}$ Isgaard and $\mathrm{H}$ Hagberg. (2005). IGF-I neuroprotection in the immature brain after hypoxia-ischemia, involvement of Akt and GSK3beta? Eur J Neurosci 21:1489-1502.

52. Arroba AI, D Wallace, A Mackey, EJ de la Rosa and TG Cotter. (2009). IGF-I maintains calpastatin expression and attenuates apoptosis in several models of photoreceptor cell death. Eur J Neurosci 30:975-986.

53. Kooijman R, S Sarre, Y Michotte and J De Keyser. (2009). Insulin-like growth factor I: a potential neuroprotective compound for the treatment of acute ischemic stroke? Stroke 40:e83-e88.

54. Iwanuma O, S Abe, E Hiroki, S Kado, K Sakiyama, A Usami and Y Ide. (2008). Effects of mechanical stretching on caspase and IGF-1 expression during the proliferation process of myoblasts. Zoolog Sci 25:242-247.

55. Jimenez Del Rio M and C Velez-Pardo. (2006). Insulin-like growth factor-1 prevents Abeta[25-35]/(H2O2)-induced apoptosis in lymphocytes by reciprocal NF-kappaB activation and p53 inhibition via PI3K-dependent pathway. Growth Factors 24:67-78.

56. Bogaert E, P Van Damme, K Poesen, J Dhondt, N Hersmus, D Kiraly, W Scheveneels, W Robberecht and L Van Den Bosch. (2009). VEGF protects motor neurons against excitotoxicity by upregulation of GluR2. Neurobiol Aging. [Epub ahead of print].

57. Van Damme P, D Braeken, G Callewaert, W Robberecht and L Van Den Bosch. (2005). GluR2 deficiency accelerates motor 
neuron degeneration in a mouse model of amyotrophic lateral sclerosis. J Neuropathol Exp Neurol 64:605-612.

58. Van Damme P, E Bogaert, M Dewil, N Hersmus, D Kiraly, W Scheveneels, I Bockx, D Braeken, N Verpoorten, K Verhoeven, V Timmerman, P Herijgers, G Callewaert, P Carmeliet, L Van Den Bosch and W Robberecht. (2007). Astrocytes regulate GluR2 expression in motor neurons and their vulnerability to excitotoxicity. Proc Natl Acad Sci U S A 104:14825-14830.

59. Van Damme P, L Van Den Bosch, E Van Houtte, G Callewaert and W Robberecht. (2002). GluR2-dependent properties of AMPA receptors determine the selective vulnerability of motor neurons to excitotoxicity. J Neurophysiol 88:1279-1287.
Address correspondence to: Dr. Eva L. Feldman Department of Neurology University of Michigan 5017 BSRB

109 Zina Pitcher Place Ann Arbor, MI 48109

E-mail: efeldman@med.umich.edu

Received for publication January 4, 2010

Accepted after revision April 20, 2010

Prepublished on Liebert Instant Online April 20, 2010 
